\title{
SHORT COMMUNICATION Sodium channel $\beta 1$-subunit expression is increased in reactive astrocytes in a rat model for mesial temporal lobe epilepsy
}

\author{
Jan A. Gorter, ${ }^{1,2}$ Erwin A. van Vliet, ${ }^{1}$ Fernando H. Lopes da Silva, ${ }^{1,2}$ Lori L. Isom ${ }^{3}$ and Eleonora Aronica ${ }^{2,4}$ \\ ${ }^{1}$ Swammerdam Institute for Life Sciences, Section of Neurobiology, University of Amsterdam, Kruislaan 320, \\ 1098 SM Amsterdam, The Netherlands \\ ${ }^{2}$ Stichting Epilepsie Instellingen Nederland, Heemstede, The Netherlands \\ ${ }^{3}$ Department of Pharmacology, The University of Michigan, Ann Arbor, MI 48109-0632, USA \\ ${ }^{4}$ Department of (Neuro)Pathology, Academic Medical Center, University of Amsterdam, The Netherlands
}

Keywords: immunocytochemistry, neurons, glia, status epilepticus, hippocampus

\begin{abstract}
As several epilepsy syndromes are associated with changes in sodium channel subunits we investigated the expression of $\beta 1$ sodium channel protein in a rat epilepsy model. In this model a chronic epileptic syndrome develops after electrically induced status epilepticus (SE). Many neuropathological characteristics of mesial temporal lobe epilepsy can be reproduced (cell loss, gliosis and synaptic reorganization). In control hippocampus $\beta 1$ subunit protein was moderately expressed in neurons and weakly expressed in resting astrocytes. $\beta 1$ sodium channel immunoreactivity increased markedly within 1 week after SE mainly in astrocytes that were colocalized with vimentin (marker for reactive astrocytes). This up-regulation was still present in reactive astrocytes of chronic epileptic rats ( $>3$ months after SE). Considering the fact that the $\beta 1$ subunits may function as cell adhesion molecules interacting with extracellular matrix, the observed increase in reactive astrocytes might subserve a function in cellular and synaptic reorganization during epileptogenesis.
\end{abstract}

\section{Introduction}

Voltage-gated sodium channels (NaChs) consist of a complex of different glycosylated subunits, including a pore-forming $\alpha$-subunit of $260 \mathrm{kDa}$ and three $\beta$-subunits of $36 \mathrm{kDa}\left(\beta_{1}\right), 33 \mathrm{kDa}\left(\beta_{2}\right)$ and the recently identified $\beta_{3}$ subunit (Catterall, 1995; Catterall, 1999; Morgan et al., 2000). The $\alpha$-subunit has been shown to be both necessary and sufficient for the formation of a functional channel (Moorman et al., 1990). The $\beta$-subunits do not form the pore, but play critical roles in modulating the activation and inactivation kinetics of sodium current and the channel expression levels (Patton et al., 1994; Isom et al., 1995b; McCormick et al., 1998; Morgan et al., 2000). In addition, it has been shown recently, that $\beta$-subunits may function as cell adhesion molecules, interacting with extracellular matrix proteins and representing a critical communication link between extracellular signalling molecules and the cytoskeleton (Isom, 2001; Isom et al., 1995a; Xiao et al., 1999; Malhotra et al., 2000).

Several lines of evidence underscore a possible role of $\mathrm{NaChs}$ in epilepsy. Firstly, we have recently shown permanent changes in neonatal Nav1.2 and Nav1.3 $\alpha$ isoforms in hippocampal neurons (Aronica et al., 2001) after electrically induced SE in the rat, associated with altered electrophysiological properties (Ketelaars et al., 2001). Moreover, mutations in $\mathrm{NaCh} \alpha$ and $\beta$ subunit genes

Correspondence: Dr Jan A. Gorter, ${ }^{1}$ Swammerdam Institute for Life Sciences, as above.

E-mail: gorter@science.uva.nl

Received 25 February 2002, revised 13 May 2002, accepted 20 May 2002 have been linked to specific epileptic syndromes. The potential clinical importance of $\mathrm{NaCh} \beta 1$ subunits in epilepsy is underscored by the identification of a point mutation in the $\beta 1$ subunit in human GEFS+ (generalized epilepsy with febrile seizures plus) (Wallace et al., 1998). This led us to investigate the expression patterns of the $\mathrm{NaCh} \beta 1$-subunit after electrically induced SE in the rat. By studying the $\beta 1$ protein expression at different phases of the epileptogenic process in this experimental model for mesial temporal lobe epilepsy, we hope to get insight in their role to hippocampal excitability and/or synaptic reorganization.

\section{Materials and methods}

\section{Experimental animals}

Male Sprague-Dawley rats (Harlan CPB laboratories, The Netherlands) weighing 400 gram at the time of the experiment were used in this study. The rats were housed in individual cages under a controlled environment $\left(21 \pm 1{ }^{\circ} \mathrm{C}\right.$; humidity $60 \%$; lights on 08.00-20.00 h). Food and water were available ad libitum. The procedures of electrode implantation, seizure induction, continuous EEG measurements using seizure detection software have been extensively described elsewhere (Gorter et al., 2001). In short, the rats underwent a series of tetanic stimulations $(50 \mathrm{~Hz})$ of the hippocampus in the form of a succession of trains of pulses every $13 \mathrm{~s}$. Each train had a duration of $10 \mathrm{~s}$ and consisted of biphasic pulses (pulse duration $0.5 \mathrm{~ms}$, maximal intensity $500 \mu \mathrm{A}$ ). 

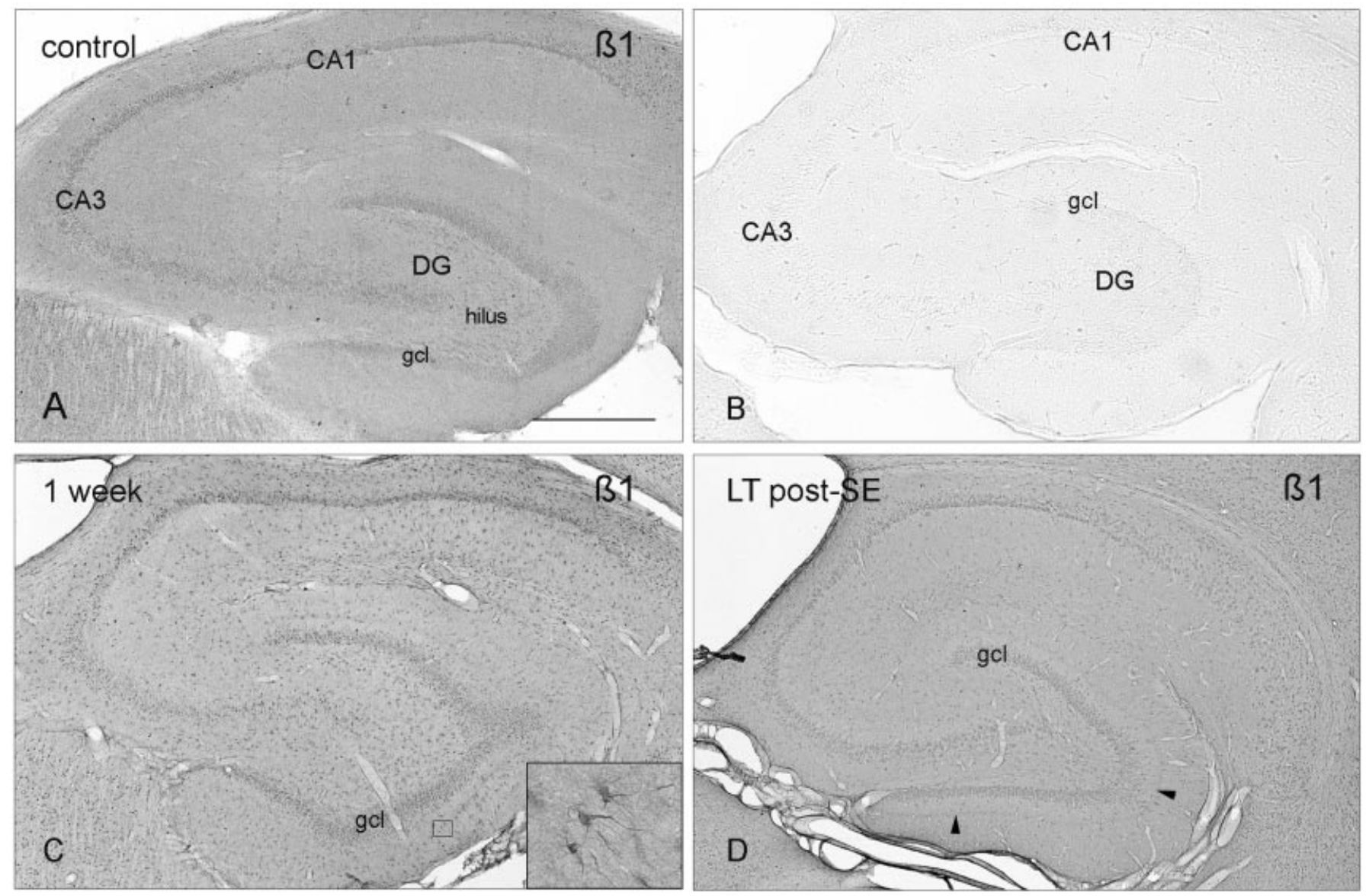

FIG 1 Electrically induced status epilepticus increased $\mathrm{NaCh} \beta 1$ subunit protein expression. (A, C and D) Representative photomicrographs of NaCh $\beta 1$ IR in the hippocampus of control (A), 1 week (C), and 4 months (chronic epileptic phase; D) post-SE. NaCh $\beta 1$ labelling was observed in neurons of the CA fields and dentate gyrus (DG) including hilar neurons. NaCh $\beta 1$ IR was rarely seen in resting glial cells in control hippocampus (A). Increased $\beta 1$ immunoreactivity was detected 1 week $(\mathrm{C})$, in the DG and CA fields where major reactive astrocytosis occurred. At 4 months post-SE the increased staining for NaCh $\beta 1$ subunit in glial cells was observed, particularly in the hilar region (see also Fig. 2D-F) and at the outer aspect of the inner molecular layer (arrow-heads) of the dentate gyrus and in stratum lacunosum moleculare. The insets in $\mathrm{C}$ show detail of intensively labelled cells with astroglial morphology (indicated by rectangle). (B) Control section incubated without the primary Ab. (A-D) Calibration bar, $500 \mu \mathrm{m}(\mathrm{A})$.

Stimulation was stopped when the rats displayed sustained forelimb clonus and strong salivation for minutes. This behavioural condition was usually reached within $1 \mathrm{~h}$. In this way a state characterized by limbic electrographic seizure activity that we call here status epilepticus (SE) was induced. Sham-operated rats $(n=3)$ and nonoperated rats $(n=2)$ served as controls. The experimental protocols were approved by the University animal welfare committee. Control and experimental rats were anaesthetized with pentobarbital and perfusion-fixed through the aorta with $4 \%$ paraformaldehyde in $0.1 \mathrm{M}$ phosphate buffer and $0.2 \%$ glutaraldehyde $(\mathrm{pH} \mathrm{7.4)}$ at different time points after stimulation: $24 \mathrm{~h}(n=3 \mathrm{SE}), 1$ week $(n=4 \mathrm{SE})$ and $3-$ 5 months $(n=5$ LT-SE). The LT-SE rats used in this study had a latent period of at average 8-10 days and all experienced frequent spontaneous seizures (on average approximately ten seizures per day) at this time point (Gorter et al., 2001). After one night in situ postfixation, the brains were dissected and transferred to $30 \%$ sucrose in $0.1 \mathrm{M}$ phosphate buffer. After an overnight incubation at $4{ }^{\circ} \mathrm{C}$, $40 \mu \mathrm{m}$ thick sections were cut in the horizontal plane on a sliding microtome and processed for immunocytochemistry.

\section{Immunocytochemistry}

Immunocytochemistry was performed with a polyclonal antibody (anti $\beta 1_{\mathrm{ex}} ; 1: 150$ dilution) generated against the extracellular domain of $\mathrm{NaCh} \beta 1$ subunit (KRRSETTAETFTEWTFR; Xiao et al., 1999). For double labelling we used the anti-glial fibrillary acidic protein (GFAP; monoclonal mouse, Boehringer Mannheim, Germany, 1 : 500), anti-vimentin (DAKO, Denmark; mouse clone V9; $1: 25$ ), and the anti-rat CD11b/c (monoclonal mouse, OX42; Pharmingen, CA, USA; $1: 100$, as marker for microglia). Single- and double-label immunocytochemistry was performed on free-floating sections. Single labelling was carried out using avidin-biotin peroxidase method (Vector Elite, CA, USA) and 3,3'-diaminobenzidine (DAB) as a chromogen. For the double labelling, sections (after the incubation with the primary $A b$ ), were incubated for $2 \mathrm{~h}$ with a $1: 200$ dilution of ALEXA 488 goat anti-mouse IgG antisera (Molecular probes, Eugene, USA) and CY3 goat anti-rabbit IgG antisera (Zymed, CA, USA). Sections were analyzed by means of a laser scanning confocal microscope (Biorad, MRC1024) equipped with argon-ion laser. Control sections incubated without the primary $\mathrm{Ab}$ or replacing it with preimmune sera were essentially blank (Fig. 1B).

\section{Evaluation of immunostaining}

Using a light microscope all sections were examined by two observers independently. The intensity, the cellular localization, and the frequency of immunoreactive glial cells were examined in 

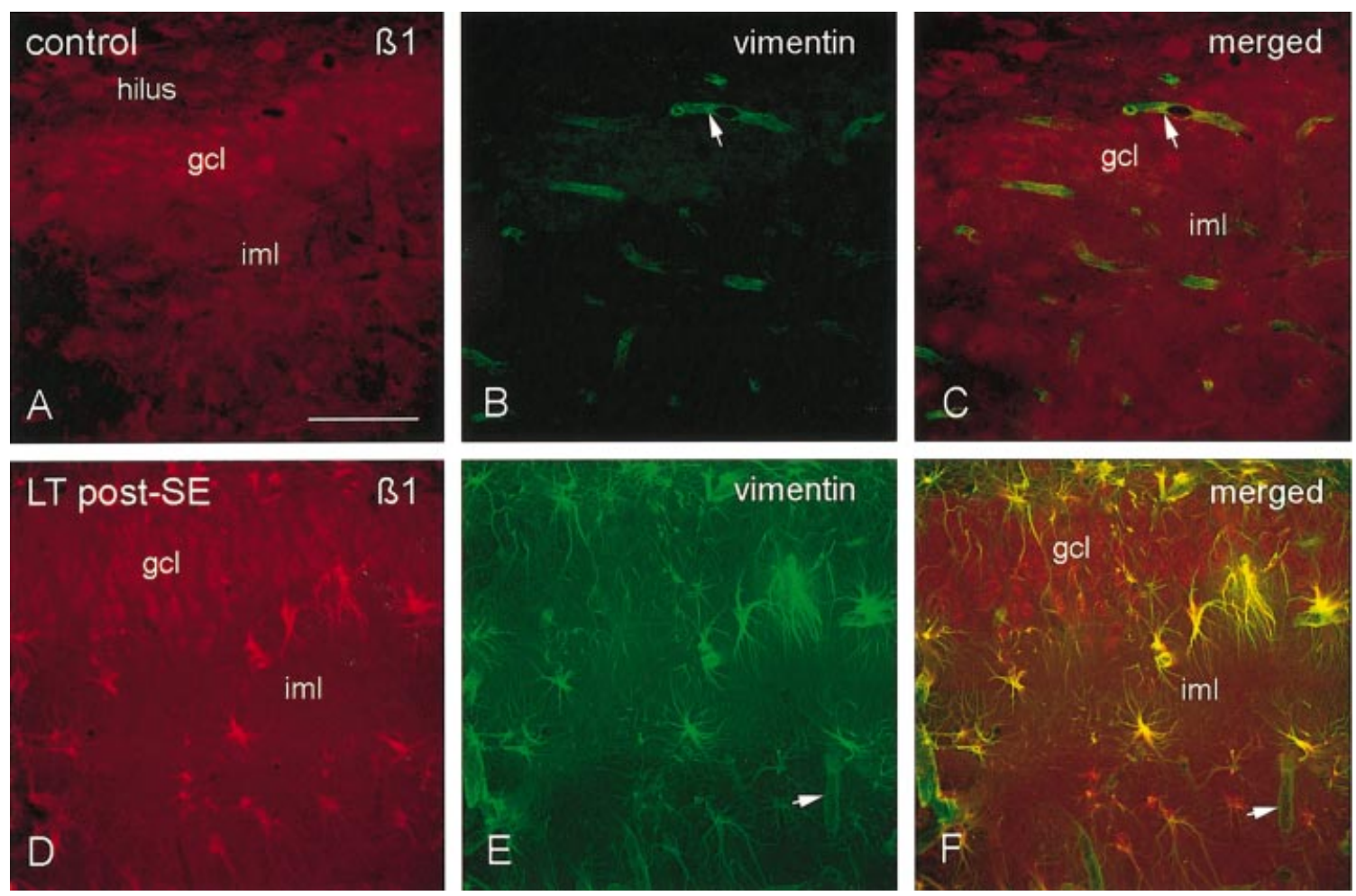

FIG 2 Confocal laser scanning microscopic images of $\beta 1$ subunit positive cells (red) and vimentin (green) in the dentate gyrus of control hippocampus (A-C) and at 4 months after electrically induced SE (D-F). (C) Merged image of control tissue indicating moderate $\beta 1$ neuronal staining in hilar and granule cell neurons. Only blood vessels are vimentin-positive (arrows indicate examples of vessels). (F) Merged image of LT-SE tissue shows that vimentin-positive reactive astrocytes display strong $\beta 1$ subunit IR (yellow). Arrow indicates blood vessel. Iml, inner molecular layer; gcl, granule cell layer. (A-F) Calibration bar, $90 \mu \mathrm{m}(\mathrm{A})$.

TABLE 1. Distribution of $\mathrm{NaCh} \beta 1$-subunit protein immunoreactivity in astroglial cells after induction of status epilepticus in rat hippocampus

\begin{tabular}{lllll}
\hline & CA1 & CA3 & DG IML & DG hilus \\
\hline Control & - & - & \pm & \pm \\
$24 \mathrm{~h}$ & $\pm(1)$ & $\pm(1)$ & $\pm(2)$ & $\pm(2)$ \\
1 week post-SE & $\pm(3)$ & $++(3)$ & $+++(3-4)$ & $+++(3-4)$ \\
$>3$ months post-SE & $+(1-2)$ & $+(2)$ & $++(2)$ & $++(2)$ \\
\hline
\end{tabular}

DG, dentate gyrus; IML, inner molecular layer. Immunoreactivity in glial cells: +++ , very intense; ++ , intense; + , positive; \pm just above background; - not detectable. Frequency scores of immunopositive cells with astroglia morphology: (1) rare; (2) moderate; (3), high; (4), very high. The frequency scores correspond with frequency of vimentin positive cells (Aronica et al., 2000).

different hippocampal regions (CA1, CA3, dentate gyrus) of control rats and rats killed at different time points after induction of SE. The immunostaining was rated and a consensus score was obtained. We rated the degree of staining and the frequency of immunopositive glial cells on a semi-quantitative point scale as indicated in Table 1.

\section{Western blot analysis}

Western blot analysis was performed on samples of the dentate gyrus (DG) region, micro dissected from the hippocampus of controls rats $(n=5)$ and rats killed 1 week $(n=5)$ and 3 months $(n=3)$ after electrically induced SE. Samples were homogenized in lysis buffer containing $10 \mathrm{~mm}$ Tris (pH 8.0), $150 \mathrm{~mm} \mathrm{NaCl}, 10 \%$ glycerol, $1 \%$ $\mathrm{NP}-40,5 \mathrm{~mm}$ ethylenediamine tetra-acetic acid (EDTA) and protease inhibitor cocktail (Boehringer). Protein content was determined by using the bicinchoninic acid method (Smith et al., 1985). Samples were diluted to a concentration of $2 \mathrm{mg}$ protein/ml in SDS/ bromophenol blue loading buffer, and boiled for $5 \mathrm{~min}$ for electrophoresis, equal amounts of proteins were subjected to SDSpolyacrylamide gel electrophoretic analysis. Separated proteins were transferred to nitrocellulose paper for $1 \mathrm{~h}$, using a semi-dry electroblotting system (Bio-Rad, Transblot SD), and incubated in TTBS (50 mM Tris-HCl, 0.1\% Tween-20, $154 \mathrm{~mm} \mathrm{NaCl,} \mathrm{pH} \mathrm{7.5),}$ containing $5 \%$ non fat dry milk and $1 \%$ bovine serum albumin (BSA) for $1 \mathrm{~h}$. Samples were then incubated over night in TTBS : 3\% BSA : $0.1 \%$ sodium azide, containing the primary antibody (anti$\beta 1_{\mathrm{ex}}$, polyclonal rabbit $(1: 1500)$; anti-actin, monoclonal mouse, Sigma, St. Louis, MO, $1: 1000)$. After several washes in TTBS, the membranes were incubated in TTBS : $5 \%$ non fat dry milk : $1 \%$ BSA, containing the goat anti-rabbit or anti-mouse coupled to horse radish peroxidase ( $1: 1500$; DAKO) for $2 \mathrm{~h}$. Immunoreactive bands were visualized using an enhanced chemiluminescence kit (ECL, Amersham). The levels of $\beta 1$ protein were evaluated by measuring optical densities of the protein bands using Scion Image for Windows (beta 4.02) image-analysis software. Expression of $\beta$-actin (as reference protein) in the same protein extracts did not change.

\section{Results}

Immunocytochemical analysis in control rat hippocampus showed $\mathrm{NaCh} \beta 1$ immunoreactivity (IR) in the principal neurons of the CA fields and in the dentate gyrus (DG) as well as in interneurons (Fig. 1A). NaCh $\beta 1$ IR was not easily detected in resting glial cells; using the more sensitive fluorescent immunostaining, however, a 

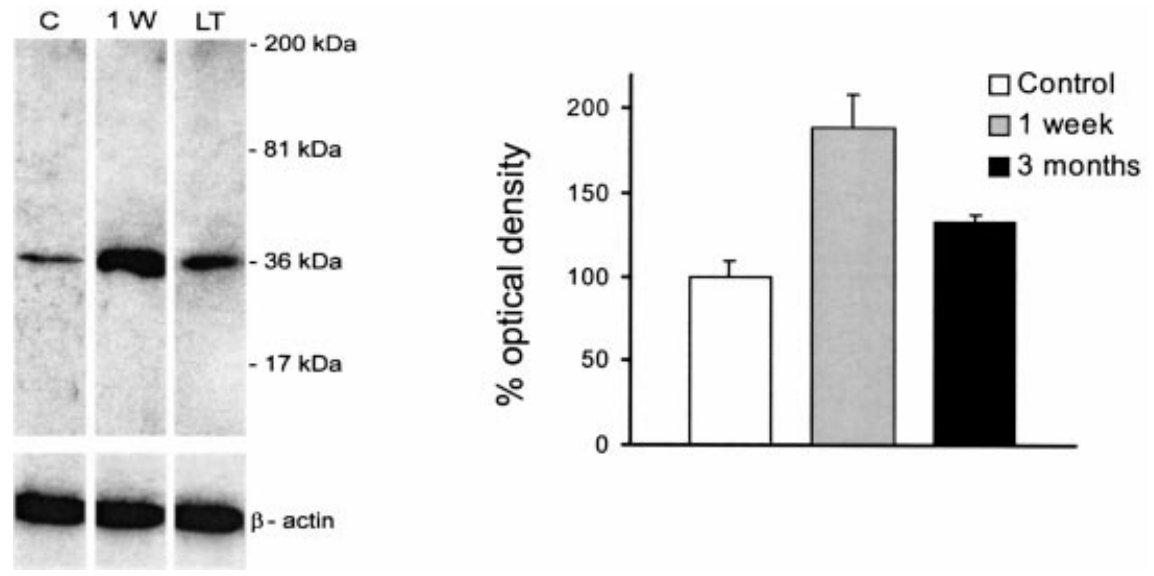

FIG 3 (A) Representative immunoblot of $\mathrm{NaCh} \beta 1$ subunit in total homogenates of the micro-dissected dentate gyrus (DG) of control rat and of a rat sacrificed 1 week and 3 months post-SE, respectively. Proteins $(20 \mu \mathrm{g} / \mathrm{lane})$ were subjected to Western blot analysis with specific antibody. $\beta$-actin expression from the same protein extracts is shown as reference protein. (B)Optical density of $\mathrm{NaCh} \beta 1$ subunit protein band of control $(n=5)$ and post-SE rats in the DG at 1 week $(n=5)$ and 3 months $(n=3)$ after SE. Values are expressed relative to control expression $(=100 \%) \pm$ SEM

weak staining in some cells with astrocyte morphology was observed (Fig. 2A). Co-localization with GFAP (data not shown) confirmed the expression in astrocytes

Electrically induced SE produced persistent changes in $\mathrm{NaCh} \beta 1$ IR in glial cells. The increased expression of $\beta 1$ subunit was first observed in 1-week post-SE tissue and persisted up to $3-5$ months post-SE (Fig. 1C and D; Table 1). At 3-5 months post-SE the increased staining for $\beta 1$ subunit in astrocytes was still observed, although it was less intense than observed at 1 week. This was particularly visible in the hilar region and inner molecular layer of the DG (Fig. 2E and F) where gliosis, visualized by vimentin-positive reactive astrocytes, persists (Aronica et al., 2000). Also an increased staining was found at the border between the inner and middle molecular layer. Western blotting, performed on samples of the DG confirmed the increased expression of the protein following SE (Fig. 3A). $\beta 1$ expression was high at one-week post-SE in the DG in all samples. At three months post-SE, $\beta 1$ expression was still high, but much lower than at one-week post-SE (Fig. 3B). The frequency of $\beta 1$ positive astrocytes varied from rat to rat and was related to the frequency of vimentin stained astrocytes (Table 1). Outside the hippocampus, we also noted $\beta 1$-positive astrocytes in the entorhinal cortex of post-SE rats, in particular in layer III. The frequency of $\beta 1$ positive astrocytes in this region was rare-moderate. Using DAB as chromogen we did not observe $\beta 1$-positive astrocytes in controls

Fluorescent microscopic analysis showed colocalization of $\beta 1$ subunit with vimentin (Fig. $2 \mathrm{~F}$ ) in reactive astrocytes. A double labelling experiment with the microglia indicator OX42 did not show expression of $\beta 1$ subunit in activated microglial cells (data not shown). $\mathrm{NaCh} \beta 1$ protein expression in individual neurons appeared to be unchanged but decreased expression was sometimes evident and probably related to neuronal cell loss (occurring mainly in the hilus and CA1-3 regions; Aronica et al., 2000; Gorter et al., 2001).

\section{Discussion}

The present study shows that $\mathrm{NaCh} \beta 1$ protein expression is selectively up-regulated in reactive astrocytes while $\beta 1$ expression in the surviving neurons appeared to be unchanged after status epilepticus. A known property of astrocytes is the morphological transformation that occurs following electrically induced SE, characterized by hypertrophy, and strong de novo immunoreactivity to vimentin. Initially, this reaction is generalized throughout the hippocampus, whereas the protracted reaction (observed up to $3-$ 5 months after SE) is more specifically localized in those regions where neuronal cell loss occurs (Aronica et al., 2000; Gorter et al., 2001). The increase of $\beta 1$ staining was clearly present throughout the hippocampus at the end of the latent period (1 week post-SE) and interestingly, it persisted in the chronic stage of epilepsy in the hypertrophic vimentin-positive astrocytes (e.g. in hilar region and inner molecular layer of DG).

$\mathrm{NaCh} \beta$ subunits are known to modulate channel gating and channel expression levels at the plasma membrane (for review see Isom, 2001). $\mathrm{NaCh} \beta 1$ subunits modulate the gating properties of the channel-forming $\alpha$ subunits, resulting in an acceleration of inactivation (Mccormick et al., 1998). Interestingly, $\mathrm{NaChs}$ have been observed in astrocytes from epilepsy biopsies and, unlike resting astrocytes, these seizure-associated astrocytes expressed $\mathrm{NaChs}$ at high densities, sufficient to generate action potential-like responses (Bordey \& Sontheimer, 1998). Whether the astrocytes in our rat model undergo similar changes in excitability remains to be determined. Considering the fact that there is a stronger $\mathrm{NaCh} \beta 1$ expression during the latent period (when rats do not have spontaneous seizures), than at the chronic epileptic phase (when the rats experience several seizures per day) suggests that the $\mathrm{NaCh} \beta 1$ upregulation might also counteract excitability. Moreover, we also observed a $\mathrm{NaCh} \beta 1$ up-regulation in reactive astrocytes obtained from patients with a brain pathology that was not associated with epilepsy (Aronica, unpublished observations). More recently, $\beta$ subunits have been shown to function as cell adhesion molecules, interacting with extracellular matrix molecules (such as tenascin-R), with cytoskeletal elements or other cell adhesion molecules (such as contactin) and being involved in the regulation of cell migration and cellular aggregation (for review see Isom, 2001). The role of $\beta 1$ subunits as cell adhesion molecules, and in particular their interaction with tenascin-R, which is known to play a role in neuronal network reorganization after injury (Faissner, 1997), is particularly interesting in post-SE hippocampus. The persistent increase of $\mathrm{NaCh} \beta 1$ protein expression in reactive astrocytes, as observed in the hilar region and inner molecular layer, could play a critical role in the patterning of mossy fibre sprouting that already starts in the latent period 
(Hendriksen et al., 2001), as well as the granule cell dispersion that can be observed in the chronic epileptic phase. Both phenomena are characteristic features of mesial temporal lobe epilepsy.

In summary, we conclude that the observed $\mathrm{NaCh} \beta 1$ subunit upregulation may represent a novel mechanism for modulation of glial function and for changes in glial-neuronal communication in epilepsy. Further studies will be necessary to get a better understanding of the possible involvement of the $\mathrm{NaCh} \beta 1$ subunits in synaptic reorganization and/or hyperexcitability that occurs in the epileptic brain. Moreover as $\mathrm{NaChs}$ are also targets for many antiepileptic drugs, the relationship between $\beta 1$ subunits and drug resistance (Catterall, 1999) also needs to be explored.

\section{Acknowledgements}

This work was supported by the National Epilepsy Fund - 'Power of the Small' (NEF20-03; J.A. Gorter and E.A van Vliet; NEF 02-10; E. Aronica) and by the NSF IBN-9734462 to L.L. Isom. We thank W.P. Meun for expert photography.

\section{Abbreviations}

DAB, 3,3'-diaminobenzidine; DG, dentate gyrus; GFAP, anti-glial fibrillary acidic protein; IR, immunoreactivity; $\mathrm{NaCh}$, voltage gated sodium channel; SE, status epilepticus.

\section{References}

Aronica, E., van Vliet, E.A., Mayboroda, O.A., Troost, D., da Silva, F.H. \& Gorter, J.A. (2000) Upregulation of metabotropic glutamate receptor subtype mGluR3 and mGluR5 in reactive astrocytes in a rat model of mesial temporal lobe epilepsy. Eur. J. Neurosci., 12, 2333-2344.

Aronica, E., Yankaya, B., Troost, D., van Vliet, E.A., Lopes da Silva, F.H. \& Gorter, J.A. (2001) Induction of neonatal sodium channel II and III alphaisoform mRNAs in neurons and microglia after status epilepticus in the rat hippocampus. Eur. J. Neurosci., 13, 1261-1266.

Bordey, A. \& Sontheimer, H. (1998) Properties of human glial cells associated with epileptic seizure foci. Epilepsy Res., 32, 286-303.

Catterall, W.A. (1995) Structure and function of voltage-gated ion channels. Annu. Rev. Biochem., 64, 493-531.

Catterall, W.A. (1999) Molecular properties of brain sodium channels: an important target for anticonvulsant drugs. Adv. Neurol., 79, 441-456.

Faissner, A. (1997) The tenascin gene family in axon growth and guidance. Cell Tissue Res., 290, 331-341.

Gorter, J.A., van Vliet, E.A., Aronica, E. \& Lopes da Silva, F.H. (2001)
Progression of spontaneous seizures after status epilepticus is related with extensive bilateral loss of hilar parvalbumin and somatostatin immunoreactive neurons. Eur. J. Neurosci., 13, 1-14.

Hendriksen, H., Datson, N.A., Ghijsen, W.E.J.M., van Vliet, E.A., Lopes da Silva, F.H., Gorter, J.A. \& Vreugdenhil, E. (2001) Altered hippocampal gene expression prior to the onset of spontaneous seizures in the rat post status epilepticus model. Eur. J. Neurosci., 14, 1475-1484.

Isom, L.L. (2001) Sodium channel beta subunits: anything but auxiliary. Neuroscientist, 7, 42-54.

Isom, L.L., Ragsdale, D.S., De Jongh, K.S., Westenbroek, R.E., Reber, B.F., Scheuer, T. \& Catterall, W.A. (1995a) Structure and function of the beta 2 subunit of brain sodium channels, a transmembrane glycoprotein with a CAM motif. Cell, 83, 433-442.

Isom, L.L., Scheuer, T., Brownstein, A.B., Ragsdale, D.S., Murphy, B.J. \& Catterall, W.A. (1995b) Functional co-expression of the beta 1 and type IIA alpha subunits of sodium channels in a mammalian cell line. J. Biol. Chem., 270, 3306-3312.

Ketelaars, S.O., Gorter, J.A., van Vliet, E.A., Lopes da Silva, F.H. \& Wadman, W.J. (2001) Sodium currents in isolated rat CA1 pyramidal and dentate granule neurones in the post-status epilepticus model of epilepsy. Neuroscience, 105, 109-120.

Malhotra, J.D., Kazen-Gillespie, K., Hortsch, M. \& Isom, L.L. (2000) Sodium channel beta subunits mediate homophilic cell adhesion and recruit ankyrin to points of cell-cell contact. J. Biol. Chem., 275, 11383-11388.

McCormick, K.A., Isom, L.L., Ragsdale, D., Smith, D., Scheuer, T. \& Catterall, W.A. (1998) Molecular determinants of Na+ channel function in the extracellular domain of the beta1 subunit. J. Biol. Chem., 273, 39543962 .

Moorman, J.R., Kirsch, G.E., VanDongen, A.M., Joho, R.H. \& Brown, A.M. (1990) Fast and slow gating of sodium channels encoded by a single mRNA. Neuron, 4, 243-252.

Morgan, K., Stevens, E.B., Shah, B., Cox, P.J., Dixon, A.K., Lee, K., Pinnock, R.D., Hughes, J., Richardson, P.J., Mizuguchi, K. \& Jackson, A.P. (2000) Beta 3: an additional auxiliary subunit of the voltage-sensitive sodium channel that modulates channel gating with distinct kinetics. Proc. Natl. Acad. Sci. USA, 97, 2308-2313.

Patton, D.E., Isom, L.L., Catterall, W.A. \& Goldin, A.L. (1994) The adult rat brain beta 1 subunit modifies activation and inactivation gating of multiple sodium channel alpha subunits. J. Biol. Chem., 269, 17649-17655.

Smith, P.K., Krohn, R.I., Hermanson, G.T., Mallia, A.K., Gartner, F.H., Provenzano, M.D., Fujimoto, E.K., Goeke, N.M., Olson, B.J. \& Klenk, D.C. (1985) Measurement of protein using bicinchoninic acid. Anal. Biochem., 150, 76-85.

Wallace, R.H., Wang, D.W., Singh, R., Scheffer, I.E., George, A.L. Jr, Phillips, H.A., Saar, K., Reis, A., Johnson, E.W., Sutherland, G.R., Berkovic, S.F. \& Mulley, J.C. (1998) Febrile seizures and generalized epilepsy associated with a mutation in the $\mathrm{Na}+$-channel beta1 subunit gene SCN1B. Nature Genet., 19, 366-370.

Xiao, Z.C., Ragsdale, D.S., Malhotra, J.D., Mattei, L.N., Braun, P.E., Schachner, M. \& Isom, L.L. (1999) Tenascin-R. is a functional modulator of sodium channel beta subunits. J. Biol. Chem., 274, 26511-26517. 